\title{
UNIVERSAL SPACES UNDER STRONG HOMEOMORPHISMS $\left({ }^{(1)}\right.$
}

BY

\section{S. W. HAHN}

1. Introduction. Let $E_{n}$ denote a Euclidean space of $n$ dimensions. We make frequent use of certain subsets of $E_{n}$ which are denoted by $R_{n}^{j}, M_{n}^{j}$, and $L_{n}^{j}$. These sets consist of the totality of points in $E_{n}$ with exactly, at most, and at least $j$ rational coordinates, respectively, and their dimensions are $0, j$, and $n-j$, in that order [4, pp. 19,42](2). If $K$ is a subset of $E_{n}$, its complement in $E_{n}$ will be designated by $C(K)$. Throughout the paper $n$ will represent an arbitrary but fixed positive integer.

If $A$ and $B$ are subsets of $E_{n}$, then $A$ will be called strongly homeomorphic to $B$ if there exists a homeomorphism (in the ordinary sense) $h$ of $E_{n}$ onto itself such that $h(A)=B$. A subset $X$ of $E_{n}$ will be called universal under strong homeomorphisms for $k$-dimensional subsets of $E_{n}(0 \leqq k \leqq n)$ if : (1) $\operatorname{dim} X$ $=k$; (2) every $k$-dimensional subset $Y$ of $E_{n}$ is strongly homeomorphic to a subset of $X$. In the sequel "universal" will always mean "universal under strong homeomorphisms."

Hurewicz and Wallman [4, p. 65] remark, "It is not known . . . whether there exists any $k$-dimensional subset of $E_{n}$ containing a topological image of every $k$-dimensional subset of $E_{n}$ " when $n<2 k+1$. The problem under consideration in this paper is related to that suggested by Hurewicz and Wallman, namely: do there exist universal $k$-dimensional subsets of $E_{n}$ for $0 \leqq k$ $\leqq n$ ? It is proved that for $k=n$ and for $k=n-1$ such universal sets do exist, while for $k \leqq n-2$ the answer is in the negative.

The results obtained suggest similar problems. Do there exist $k$-dimensional subsets of $E_{n}$ which contain strongly homeomorphic images of all members of a given class (for example, the class of all compact $k$-dimensional subsets of $E_{n}$ ) if $k \leqq n-2$ ? Are there $j$-dimensional subsets of $E_{n}$ which contain strongly homeomorphic images of every $k$-dimensional subset of $E_{n}$, where $k<j<n-1$ ? In the last section we investigate briefly one such problem.

The author wishes to thank Professor J. H. Roberts for his suggestion of the problem and for his invaluable assistance in the preparation of this paper.

2. THEOREM 2. For $k=n$ and for $k=n-1$ the set $M_{n}^{k}$ is universal under strong homeomorphisms for k-dimensional subsets of $E_{n}$.

Presented to the Society, September 5, 1947; received by the editors June 26, 1950 .

(1) This paper is taken from a thesis submitted in partial fulfillment of the requirements for the degree of Doctor of Philosophy at Duke University.

(2) Numbers in brackets refer to the bibliography at the end of the paper. 
Proof. The theorem is trivial in the case $k=n$, since $M_{n}^{n}=E_{n}$.

Let $Y$ denote an arbitrary $(n-1)$-dimensional point set in $E_{n}$. Then $C(Y)$ is dense in $E_{n}[4, \mathrm{p} .44]$. Hence $C(Y)$ contains a countable subset $A$ which is also dense in $E_{n}$. Likewise $C\left(M_{n}^{n-1}\right)=R_{n}^{n}$ is countable and dense in $E_{n}$. Then there exists a homeomorphism $h$ of $E_{n}$ onto itself such that $h(A)=R_{n}^{n}[3, \mathrm{p}$. 159]. Since $A \subset C(Y)$, this gives $R_{n}^{n}=h(A) \subset h[C(Y)]=C[h(Y)]$. Passing to complementary sets, $h(Y) \subset M_{n}^{n-1}$.

Note that for all $Y \subset E_{n}$ with $\operatorname{dim} Y \leqq n-1, C(Y)$ is dense in $E_{n}$. Hence Theorem 2 can be strengthened to give the following theorem.

THEOREM $2^{\prime}$. For $k=n$ and for $k=n-1, M_{n}^{k}$ contains a strongly homeomorphic image of every subset $Y$ of $E_{n}$ for which $\operatorname{dim} Y \leqq k$.

3. Lemmas and definitions. In $\S 4$ we make repeated use of a certain type of continuum associated with a plane rectangle. This type of continuum was described by Knaster [5, p. 275, example $H_{3}$ ]. Lemma 3.1 is a statement of the properties which we shall use of Knaster's continuum.

Lemma 3.1. Let $A B C D$ be a plane rectangle with interior $W$. Then there exists a continuum $N^{*}$ having the following properties: (a) $N^{*} \cap A B=P$ and $N^{*}$ $\cap C D=Q$ are single points, distinct from $A, B, C$, and $D$; (b) $N^{*}-(P \cup Q)$ is contained in $W$; (c) no subset of $N^{*}$ is an arc; (d) $\operatorname{dim} N^{*}=1$; (e) $N^{*}$ separates $W$ into exactly two domains (connected open sets) $H$ and $K$, each of which is bounded in part by one of the two components of $A B C D-(P \cup Q)$.

Properties (a)-(c) follow from Knaster's construction and discussion of $N^{*}$. Property (d) is a consequence of (a) and (c). From (a), since $N^{*}$ is a connected point set containing two distinct points, $\operatorname{dim} N^{*} \geqq 1[4$, p. 15]. But if $\operatorname{dim} N^{*}=2, N^{*}$ must contain a domain in the plane $[4, \mathrm{p} .44]$ and hence an arc, contrary to (c). Property (e) is easily established by an elementary argument based upon the method of construction of $N^{*}$. We omit this argument.

Definition 3.2. By a Knaster continuum we shall understand a plane continuum which has the properties of the set $N^{*}$ in Lemma 3.1 with respect to some rectangle.

DEFINITION 3.3. Let $J_{1}$ and $J_{2}$ be circles with interiors $R_{1}$ and $R_{2}$, such that $R_{1} \supset J_{2} \cup R_{2}$. Then the set $R_{1}-R_{2}$ will be called the (open) circular band between $J_{1}$ and $J_{2}$. Its closure will be referred to as the corresponding closed circular band.

Let $A B C D$ denote a plane rectangle with interior $W$ and $N^{*}$ a Knaster continuum associated with $A B C D$. It is evident that there exists a continuous transformation $f$ defined over $\bar{W}$ which "bends" $\bar{W}$ into a closed circular band in such a way that (1) $f(A B)=f(D C)$; (2) $f(P)=f(Q)$, where $N^{*} \cap A B C D$ $=P \cup Q ;(3) f$ is a homeomorphism over $\bar{W}-(A B \cup D C) ;(4) f(B C)$ and $f(D A)$ are circles, with $f(B C)$ interior to $f(D A)$. 
Let $H_{1}$ and $H_{2}$ denote the two domains into which $N^{*}$ separates $W$, with the notation so chosen that $\bar{H}_{1} \supset A D$. Then $f\left(N^{*}\right)$ separates $f(W)$ into $f\left(H_{1}\right)$ and $f\left(H_{2}\right)$. Clearly $f\left(N^{*}\right)$ also separates $E_{2}$ into two domains $O_{1}$ and $O_{2}$, where $O_{1}=f\left(H_{2}\right) \cup f(B C) \cup$ interior of $f(B C)$, and $O_{2}=E_{2}-\left[f\left(N^{*}\right) \cup O_{1}\right]$. Hence $f\left(N^{*}\right)$ is a continuum which contains no arc, which separates $E_{2}$ into two domains, and which lies entirely within the open circular band between $f(D A)$ and $f(B C)$.

Now suppose that $B$ is the circular band between two arbitrary circles $J_{1}$ and $J_{2}$, where $R_{1} \supset J_{2} \cup R_{2}$. It is obvious that there is a homeomorphism $h$ of the closed band $f(\bar{W})$ onto $\bar{B}$. Then the set $N=h\left[f\left(N^{*}\right)\right]$ is a 1-dimensional subset of the open band $B$ which separates $E_{2}$ and which contains no arc.

We summarize the preceding discussion in the following lemma.

LеммA 3.4. If $J_{1}$ and $J_{2}$ are circles such that $R_{1} \supset J_{2} \cup R_{2}$, then in the band $R_{1}-\bar{R}_{2}$ there is a continuum $N$ having the following properties: (a) $N$ separates $E_{2}$ into two domains, a bounded one containing $\bar{R}_{2}$ and an unbounded one containing $E_{2}-R_{1}$; (b) $\operatorname{dim} N=1$; (c) no subset of $N$ is an arc.

Definition 3.5. A continuum having the properties of $N$ in Lemma 3.4 with respect to some pair of circles in $E_{2}$ will be called an $N$-continuum.

Consider now the collection of all circles in $E_{2}$ with rational radii whose center coordinates are both rational. Let $\left\{P_{j}\right\}, j=1,2, \cdots$, denote the collection of pairs of such circles $\left(J_{m_{2}} J_{n}\right)$ having the additional property that $R_{m} \supset J_{n} \cup R_{n}$. Applying Lemma 3.4 to each pair $P_{j}$, we can find an $N$-continuum $N_{j}$ within the band formed by the two circles of $P_{j}$. Let $N_{j}$ denote a fixed $N$-continuum associated with $P_{j}$. We now define a set $M$ by

$$
M=\bigcup_{j=1}^{\infty} N_{j} .
$$

By the sum theorem on dimension for closed sets $[4$, p. 30$], \operatorname{dim} M=1$. It is also easily seen that $M$ can contain no arc. Finally, since every point in $C(M)$ is contained within arbitrarily small pairs of concentric "rational" circles, it is likewise contained in the bounded complementary domain of an $N_{j}$ of arbitrarily small diameter, whose boundary relative to $C(M)$ is vacuous. Hence $\operatorname{dim} C(M)=0$.

Definition 3.6. By an $M$-set in a plane we understand a set in $E_{2}$ defined after the manner of the above set $M$. An $M$-set, then, is a countable sum of $N$-continua; it is 1 -dimensional; it contains no arc; and its complement in $E_{2}$ is 0-dimensional.

Definition 3.7. A rational $E_{q}$ in $E_{n}, q \leqq n$, is a $q$-space determined by $x_{i_{1}}=r_{1}, x_{i_{2}}=r_{2}, \cdots, x_{i_{n-q}}=r_{n-q}$ for some choice of $n-q$ coordinates, where the $r_{i}$ are fixed rational numbers and where a point is $E_{n}$ is denoted by $\left(x_{1}, x_{2}, \cdots, x_{n}\right)$. 
Definition 3.8. The orthogonal projection of $E_{n}$ onto a rational $E_{q}$ is the transformation $T$ which carries every point $P\left(x_{1}, \cdots, x_{n}\right)$ of $E_{n}$ into the point $T(P)$ of $E_{q}$ whose coordinates are $x_{i_{j}}=r_{j}(j \leqq n-q)$ and $x_{p}=x_{p}$ for the remaining $q$ coordinates.

Definition 3.9. If $S$ is an $s$-dimensional subset of a rational $E_{q}$ in $E_{n}$, then the totality of points in $E_{n}$ whose images under the orthogonal projection of $E_{n}$ onto $E_{q}$ lie in $S$ comprise an $(s+n-q)$-dimensional cylinder on $S$.

It is evident that a rational $E_{q}$ in $E_{n}$, for $2 \leqq q \leqq n$, can be generated by forming a $q$-dimensional cylinder upon a rational plane in $E_{n}$. In fact, for $q>2$ there is a countable infinity of rational planes which will give rise to a given $E_{q}$ in this manner. Conversely, however, a given rational plane is contained in only a finite number of rational $q$-spaces. Since every rational plane in $E_{n}$ is parallel to one of the coordinate $x_{i} x_{j}$-planes, every rational $E_{q}$ can be associated with a certain number of the coordinate planes, namely those which are parallel to one of the rational planes which can be used to generate $E_{q}$.

4. In this section we complete the solution of our main problem by constructing a certain $k$-dimensional subset $C\left(M^{*}\right)$ of $E_{n}$ and then proving that when $k \leqq n-2$, no $k$-dimensional subset of $E_{n}$ can contain strongly homeomorphic images of both $C\left(M^{*}\right)$ and $M_{n}^{k}$.

THEOREM 4.1. If $0 \leqq k \leqq n-2$, there exists no universal (under strong homeomorphisms) subset of $E_{n}$.

Proof. In each of the ${ }_{n} C_{2}$ coordinate planes of $E_{n}$, or $x_{i} x_{j}$-planes determined by $x_{p}=0(p \neq i, j)$, let $M_{i j}$ denote an $M$-set. In every rational plane parallel to the $x_{i} x_{j}$-plane there is an isometric topological image of $M_{i j}$. Upon one such image $S$ of $M_{i j}$ in each rational plane parallel to the $x_{i} x_{j}$-plane we erect an $(n-k-1)$-dimensional cylinder in each of the rational $E_{n-k}$ containing the plane in which $S$ lies. Thus for each rational plane parallel to the $x_{i} x_{j}$-plane we get ${ }_{n-2} C_{k-2}$ such $(n-k-1)$-dimensional cylinders upon an isometric image of $M_{i j}$. The sum of all these cylinders we call $M_{i j}^{*}$. Finally we put

$$
M^{*}=\underset{(i, j)}{\bigcup} M_{i j}^{*}
$$

Thus a point $P$ of $E_{n}$ is in $M^{*}$ if and only if there is at least one rational $E_{n-k}$ containing $P$ for which the orthogonal projection of that $E_{n-k}$ onto a rational plane carries $P$ into a point of the $M$-set which was chosen for that plane. Now $M^{*}$ is a countable sum of $(n-k-1)$-dimensional cylinders upon $N$-continua; hence

$$
\operatorname{dim} M^{*}=n-k-1 .
$$

We have from (2) $[4$, p. 28$]$ that 


$$
\operatorname{dim} C\left(M^{*}\right) \geqq k .
$$

We complete the proof of the theorem under the additional assumption that $\operatorname{dim} C\left(M^{*}\right) \leqq k$. Then we prove (Lemma 4.2 ) that this last inequality holds. With this additional assumption we now have

$$
\operatorname{dim} C\left(M^{*}\right)=k .
$$

Now suppose that there exists a universal $k$-dimensional subset $X$ of $E_{n}$. Then there are homeomorphisms $g$ and $h$ of $E_{n}$ onto itself such that

$$
\begin{array}{r}
g\left(M_{n}^{k}\right) \subset X, \\
h\left[C\left(M^{*}\right)\right] \subset X .
\end{array}
$$

Passing to complementary sets, (5) yields

$$
C(X) \subset g\left(L_{n}^{k+1}\right) \cap h\left(M^{*}\right) .
$$

Now the set $L_{n}^{k+1}$ is a countable sum of rational $E_{n-k-1}$ and $M^{*}$ is a countable sum of $(n-k-1)$-dimensional cylinders upon $N$-continua, each cylinder being contained in some rational $E_{n-k}$. Hence

$$
C(X) \subset \bigcup_{m=1}^{\infty} A_{m}
$$

where each $A_{m}$ is the intersection of a topological image of a rational $E_{n-k-1}$ and a topological image of an $(n-k-1)$-dimensional cylinder upon an $N$ continuum. Hence each $A_{m}$ is closed, and $\operatorname{dim} A_{m} \leqq n-k-1(m=1,2, \cdots)$.

If now for every $m, \operatorname{dim} A_{m} \leqq n-k-2$, from (7) we would have $\operatorname{dim} C(X)$ $\leqq n-k-2$, but since $C(X)$ is the complement of a $k$-dimensional set in $E_{n}$, this is impossible. Thus for at least one $A_{m}$, say $A_{p}$, we must have $\operatorname{dim} A_{p}$ $=n-k-1$.

Consider $A_{p}=L_{r} \cap N_{s}$, where $L_{r}$ is the image under $g$ of a rational $E_{n-k-1}$ and $N_{s}$ is the image under $h$ of an $(n-k-1)$-dimensional cylinder upon an $N$-continuum in a rational plane. Since $g$ is a homeomorphism over all of $E_{n}$, $g^{-1}\left(A_{p}\right)=g^{-1}\left(L_{r} \cap N_{s}\right)=g^{-1}\left(L_{r}\right) \cap g^{-1}\left(N_{s}\right)$. The set $g^{-1}\left(L_{r}\right)$ is a rational $E_{n-k-1}$ in $E_{n}$, and as an $(n-k-1)$-dimensional subset of $g^{-1}\left(L_{r}\right), g^{-1}\left(A_{p}\right)$ must contain an $(n-k-1)$-cell [4, p. 44]. Let $K$ denote such a cell in $g^{-1}\left(A_{p}\right)$. Under the homeomorphism $t=h^{-1} g, t(K)$ must be an $(n-k-1)$-cell in $h^{-1}\left(A_{p}\right)$, which is a subset of $h^{-1}\left(N_{8}\right)$. Now $h^{-1}\left(N_{s}\right)$ is an $(n-k-1)$-dimensional cylinder upon an $N$-continuum in some plane, $E_{2}^{\prime}$. Also $h^{-1}\left(N_{s}\right)$ is contained in a rational $(n-k)$-space, $E_{n-k}^{\prime}$, erected upon $E_{2}^{\prime}$. Under the orthogonal projection $T$ of $E_{n-k}^{\prime}$ onto $E_{2}^{\prime}, h^{-1}\left(N_{s}\right)$ is transformed into an $N$-continuum which contains $T[t(K)]$. As the image under a continuous transformation of a Peano continuum, $T[t(K)]$ is also a Peano continuum. The dimension of $t(K)$ can be lowered by at most $n-k-2$ under $T[4$, p. 91$]$; hence $\operatorname{dim} T[t(K)]$ 
$\geqq 1$.

Now as a Peano continuum with $\operatorname{dim} \geqq 1, T[t(K)]$ is arcwise connected, but as a subset of a plane $N$-continuum $T[t(K)]$ can contain no arc. This contradiction completes the proof of Theorem 4.1 except for the following lemma.

LEMma 4.2. $\operatorname{dim} C\left(M^{*}\right) \leqq k$.

Proof. In order to prove this lemma it will suffice to show that for every rational $E_{n-k}$ in $E_{n}, \operatorname{dim} C\left(M^{*}\right) \cap E_{n-k} \leqq 0$. For from this inequality it follows that $\operatorname{dim} C\left(M^{*}\right) \cap E_{n-k+1} \leqq 1$ for every rational $E_{n-k+1}$ since every point $P$ of $C\left(M^{*}\right) \cap E_{n-k+1}$ is contained in arbitrarily small cubes in $E_{n-k+1}$ whose faces are rational $E_{n-k}$. Similarly from this latter inequality it follows that $\operatorname{dim} C\left(M^{*}\right) \cap E_{n-k+2} \leqq 2$ for every rational $E_{n-k+2}$ in $E_{n}$. Continuing this process, after $k$ steps we arrive at the inequality $\operatorname{dim} C\left(M^{*}\right) \cap E_{n} \leqq k$.

Let now $E_{n-k}^{\prime}$ denote an arbitrary rational $E_{n-k}$ in $E_{n}$. With $E_{n-k}^{\prime}$ can be associated ${ }_{n-k} C_{2}$ coordinate $x_{i} x_{j}$-planes, those in $E_{n}$ which are parallel to a rational plane from which $E_{n-k}^{\prime}$ can be generated as an $(n-k)$-dimensional cylinder. Let $S_{1}, S_{2}, \cdots, S_{c}, c={ }_{n-k} C_{2}$, be a set of rational planes, one and only one of which is parallel to each of the coordinate planes associated with $E_{n-k}^{\prime}$ in the above manner and such that $E_{n-k}^{\prime}$ is an $(n-k)$-dimensional cylinder upon each $S_{i}$.

Let $Q$ be any point of $C\left(M^{*}\right) \cap E_{n-k}^{\prime}$ and let $U$ be any open set of $E_{n-k}^{\prime}$ containing $Q$. Then there is a cube $W$ in $E_{n-k}^{\prime}$ such that $Q \subset W \subset \bar{W} \subset U$ whose faces are rational $E_{n-k-1}$. For $i=1,2, \cdots, c$, let $W_{i}$ be the image of $W$ under the orthogonal projection $T_{i}$ of $W$ into $S_{i}$. Obviously $W_{i}$ is a nondegenerate square containing $T_{i}(Q)$ as an interior point. It is possible to find two circles $J_{i}^{\prime}$ and $J_{i}^{\prime \prime}$ interior to $W_{i}$, with rational centers and radii, which satisfy $T_{i}(Q) \subset R_{i}^{\prime \prime} \subset \bar{R}_{i}^{\prime \prime} \subset R_{i}^{\prime}$. Let $N_{i}$ denote the $N$-continuum in $S_{i}$ which was chosen for this pair of circles when $M^{*}$ was formed. Then $N_{i}$ separates $S_{i}$ into an unbounded domain and a bounded one containing $T_{i}(Q)$. Similarly the set $N_{i}^{*}=T_{i}^{-1}\left(N_{i}\right)$ separates $E_{n-k}^{\prime}$ into two domains, one of which contains the point $Q$. Let $D_{i}^{*}$ denote this latter domain of $E_{n-k}^{\prime}$. For each $i$ two coordinates of every point in $D_{i}^{*}$ are bounded, and these bounds do not exceed the bounds on the corresponding coordinates for points of the cube $W$. Hence if we put $D=\bigcap_{i=1}^{c} D_{i}^{*}$ it follows that $D$ is an open subset of $W$ and hence of $U$. The boundary of $D$ is contained in the union of the boundaries of the sets $D_{i}^{*}$ and hence in $\bigcup_{i=1}^{c} N_{i}^{*}$, each point of which is in $M^{*}$. Thus the boundary of $D$ relative to $C\left(M^{*}\right)$ is vacuous.

5. Additional theorems. As was pointed out in the introduction, Theorems 2 and 4.1 leave unsettled certain related problems. In this section we consider one such problem: do there exist $k$-dimensional subsets of $E_{n}$ which contain strongly homeomorphic images of all compact $k$-dimensional subsets of $E_{n}$, for $k \leqq n-2$ ? We settle the question here only for the case $k=0$ and 
$n=2$. In addition we prove that there is a 1-dimensional subset of $E_{3}$ which contains a strongly homeomorphic image of every compact 0-dimensional subset of $E_{3}$. These results suggest the possible existence of general theorems of which they are special cases.

Lemma 5.1. If $Y$ is any compact 0 -dimensional subset of $E_{2}, L$ is any rational line in $E_{2}$, and $\epsilon>0$ is arbitrary, then there is a homeomorphism $f$ of $E_{2}$ onto itself such that (a) $f(Y) \cap L=0$, (b) $d[P, f(P)]<\epsilon$ for every point $P$ in $E_{2}$, (c) $f(P)=P$ for every $P$ in $E_{2}$ exterior to some rectangle whose interior contains $Y \cap L$.

Outline of proof. There is obviously no loss in generality if we assume $L$ to be the $x$-axis. Since $Y$ is compact, there is a number $a>0$ such that $Y$ is contained within the vertical strip bounded by the lines $x= \pm a$. Let $R$ be the rectangle bounded by $x= \pm a$ and $y= \pm \epsilon / 4$. Further subdivide $R$ into smaller rectangles by vertical lines at a maximum distance of $\epsilon / 4$ apart. Since $\operatorname{dim} Y=0, Y$ does not separate the interior of any of these small rectangles $[4$, p. 48]. With the aid of the arcwise connectedness theorem for a connected domain $[7$, p. 86] it is easily shown that there is an arc $A$ joining the points $(-a, 0)$ and $(a, 0)$ which lies, except for end points, within the horizontal strip bounded by $L$ and the upper boundary of $R$, which intersects each of the vertical sides of the small rectangles in just one point, and which contains no point of $Y$.

It is possible now by using the Schoenflies extension theorem [9, p. 324] to define a homeomorphism of each of the small rectangles and its interior onto itself in such a way that it carries the portion of the arc $A$ lying in the rectangle into the corresponding portion of the line $L$. Furthermore these homeomorphisms may be defined so that the homeomorphisms on two adjoining rectangles agree along the common side and so that all reduce to the identity on $R$. The homeomorphism $f$ of the lemma, then, is a composite of these homeomorphisms over the small rectangles and the identity outside of $R$. Properties (a) and (c) stated in the lemma obviously hold. The only points which are moved by $f$ remain within their original sub-rectangles of $R$ and hence move at most a distance of $3 \epsilon / 4$.

The following is a part of a theorem of Roberts [8, p. 252].

Lemma 5.2. Let $\left\{\epsilon_{j}\right\}, j=1,2, \cdots$, be a sequence of positive numbers such that $\sum_{j=1}^{\infty} \epsilon_{j}$ converges. Suppose that for every $j, f_{j}$ is a homeomorphism of $E_{n}$ onto itself such that for every point $P$ in $E_{n}, d\left[P, f_{j}(P)\right]<\epsilon_{j}$. For each point let $P^{1}$ denote $f_{1}(P)$, and in general let $P^{i+1}$ denote $f_{j+1}\left(P^{j}\right)$. Let $f(P)$ denote the sequential limit point of the sequence $P^{1}, P^{2}, P^{3}, \cdots$. Then a sufficient condition that $f$ be a homeomorphism of $E_{n}$ onto itself is that for every pair $(P, Q)$ of points in $E_{n}$ there exists an integer $m$ such that $d\left(P^{m}, Q^{m}\right)>\sum_{j=m+1}^{\infty} 3 \epsilon_{j}$.

LEMMA 5.3. Let $g$ be a homeomorphism of $E_{n}$ onto itself. Let $K$ be a compact 
subset of $E_{n}$. Let $\epsilon>0$ be arbitrary. Then there exists an $\alpha>0$ such that if $P$ and $Q$ are any two points of $K$ with $d(P, Q)>\epsilon$, then $d[g(P), g(Q)]>\alpha$.

THEOREM 5.4. The set $M_{2}^{0}$ contains a strongly homeomorphic image of every point set in $E_{2}$ which is the sum of a countable number of compact 0-dimensional sets.

The proof of this theorem is analogous to the more difficult proofs of Theorems 5.5 and 5.6. It will be clear to the reader of those theorems how to proceed in the present case. Accordingly we omit the present proof.

THEOREM 5.5. The set $M_{3}^{1}$ contains a strongly homeomorphic image of every compact 0-dimensional subset of $E_{3}$.

Proof. Let $M$ be an arbitrary compact 0 -dimensional subset of $E_{3}$. We shall exhibit a homeomorphism $f$ of $E_{3}$ onto itself which carries $M$ into a subset of $M_{3}^{1}$.

We have $C\left(M_{3}^{1}\right)=L_{3}^{2}=\sum_{i=1}^{\infty} L_{i}$, where each $L_{i}$ is a rational line in $E_{3}$. Each $L_{i}$ is contained in exactly two rational planes. We pick one of these planes for each $i, i=1,2, \cdots$, and denote it by $K_{i}$.

Since $M$ is compact, there is a cube $C^{*}$ of $E_{3}$ with center at the origin whose interior contains $M$ and whose faces are rational planes.

Consider the plane $K_{1}$, which contains $L_{1}$. Put $\epsilon_{1}=1 / 3 \cdot 2$. By Lemma 5.1 there is a homeomorphism $f_{1}$ of $K_{1}$ onto itself such that (a) $f_{1}\left(M \cap K_{1}\right) \cap L_{1}$ $=0$; (b) $d\left[P, f_{1}(P)\right]<\epsilon_{1}$ for $P$ in $K_{1}$; (c) $f_{1}(P)=P$ for all points in $K_{1}$ $-\left(K_{1} \cap C^{*}\right)$. We then extend the homeomorphism $f_{1}$ to all of $E_{3}$ by mapping straight lines perpendicular to $K_{1}$ at every point $P$ in $K_{1}$ into straight lines perpendicular to $K_{1}$ at $f_{1}(P)$ in such a way that every plane parallel to $K_{1}$ is transformed under $f_{1}$ into itself. This homeomorphism then is the identity transformation except on the 3-dimensional cylinder upon $C^{*} \cap K_{1}$.

Let $C$ be the cube in $E_{3}$ containing $C^{*}$ whose faces are parallel to and at a distance of 2 from the corresponding faces of $C^{*}$. By Lemma 5.3 there is an $\alpha_{1}, 0<\alpha_{1}<1 / 2$, such that if $P$ and $Q$ are any points of $C$ for which $d(P, Q)$ $>1$, then $d\left(P^{1}, Q^{1}\right)>\alpha_{1}$, where $P^{1}=f_{1}(P)$ and $Q^{1}=f_{1}(Q)$. Moreover this inequality also holds for any two points $P$ and $Q$ in $E_{3}$ for which $d(P, Q)>1$. For suppose first that both $P$ and $Q$ lie within the cylinder upon $C \cap K_{1}$. Then if the distance between the two planes $Z_{P}$ and $Z_{Q}$ parallel to $K_{1}$ and containing $P$ and $Q$ exceeds 1 , since planes parallel to $K_{1}$ are carried into themselves under $f_{1}$, we obviously have $d\left(P^{1}, Q^{1}\right)>\alpha_{1}$. But if $d\left(Z_{P}, Z_{Q}\right) \leqq 1$, it is possible to find two planes $Z_{P}^{\prime}$ and $Z_{Q}^{\prime}$ parallel to $K_{1}$ which intersect the set $C-C^{*}$ and which satisfy $d\left(Z_{P}^{\prime}, Z_{Q}^{\prime}\right)=d\left(Z_{P}, Z_{Q}\right)$. Let $P^{*}$ and $Q^{*}$ denote those points in $Z_{P}^{\prime}$ and $Z_{Q}^{\prime}$ respectively whose coordinates differ from those of $P$ and $Q$ only in the one coordinate whose fixing determines $K_{1}$. Then $d\left(P^{*}, Q^{*}\right)$ $=d(P, Q)>1$ and $^{*} d\left(P^{* 1}, Q^{* 1}\right)=d\left(P^{1}, Q^{1}\right)$. But since $P^{*}$ and $Q^{*}$ are in $C$, we have $d\left(P^{* 1}, Q^{* 1}\right)>\alpha_{1}$. Thus if both $P$ and $Q$ lie within the cylinder upon 
$C \cap K_{1}$ and $d(P, Q)>1$, then $d\left(P^{1}, Q^{1}\right)>\alpha_{1}$.

Now suppose that one of the points, $P$ say, is not in the cylinder upon $C \cap K_{1}$ and that $d(P, Q)>1$. Then $P^{1}=P$, and the distance from $P$ to the cylinder upon $C^{*} \cap K_{1}$ is not less than 2 . If $Q$ is in this cylinder, so also is $Q^{1}$; hence $d\left(P^{1}, Q^{1}\right) \geqq 2>\alpha_{1}$. Finally, if $Q$ is not in this cylinder, $Q^{1}=Q$ and we again have $d\left(P^{1}, Q^{1}\right)>\alpha_{1}$. Thus we have shown that this last inequality holds for every pair of points in $E_{3}$ for which $d(P, Q)>1$.

Put $M^{1}=f_{1}(M)$. Since $M^{1}$ and $L_{1}$ are disjoint closed sets, with $M^{1}$ compact, $d\left(M^{1}, L_{1}\right)=d_{1}>0$.

The homeomorphism $f_{1}$ is the first of an infinite sequence to be defined. For $j>1$ put

$$
\epsilon_{j}=\frac{\min \left(\alpha_{1}, \cdots, \alpha_{j-1}, d_{1}, \cdots, d_{j-1}\right)}{3 \cdot j^{2}} .
$$

The $\alpha_{i}(i=1,2, \cdots, j-1)$ are numbers satisfying $0<\alpha_{i} \leqq 1 /(i+1)$ which have the property that $d\left(P^{i}, Q^{i}\right)>\alpha_{i}$ for all points $P, Q$ in $E_{3}$ for which $d(P, Q)>1 / i$, where $P^{i}=f_{i}\left(P^{i-1}\right)$ and $P=P^{0}$. The $d_{i}$ denote the distances $d\left(M^{i}, L_{i}\right)$, where $M^{i}=f_{i}\left(M^{i-1}\right)$ and $M=M^{0}$.

Now applying Lemma 5.1 to the rational plane $K_{j}$, we get a homeomorphism $f_{j}$ of $K_{j}$ onto itself under which (a) $f_{j}\left(M^{j-1}\right) \cap L_{j}=0$, (b) $d\left[f_{j}(P), P\right]$ $<\epsilon_{j}$ for all $P$ in $K_{j}$, and (c) $f_{j}(P)=P$ for all $P$ not in $C^{*} \cap K_{j}$. Put $d\left[f_{j}\left(M^{j-1}\right)\right.$, $\left.L_{j}\right]=d_{j}>0$. The homeomorphism $f_{j}$ is then extended to all of $E_{3}$ by transforming every plane parallel to $K_{j}$ into itself in the same manner as was done in the case of $f_{1}$. It is evident that $P^{i}=f_{j}\left(P^{j-1}\right)=P$ unless $P$ is in one of the three cylinders constructed upon intersections of $C^{*}$ with the coordinate planes.

We use Lemma 5.3 again to find an $\alpha_{j}, 0<\alpha_{j} \leqq 1 /(j+1)$, such that if $P$ and $Q$ are points of $C$ for which $d(P, Q)>1 / j$, then $d\left(P^{i}, Q^{i}\right)>\alpha_{j}$. A simple extension of the analysis previously made for $\alpha_{1}$ suffices to prove that the latter inequality holds as well for all pairs of points $P, Q$ in $E_{3}$ for which $d(P, Q)>1 / j$. Since points lying in three mutually perpendicular cylinders are now subject to possible change, it is necessary to examine more cases than before. These, however, present no difficulties, and we omit the details here.

Let $f(P)$ denote the sequential limit point of the sequence $P^{1}, P^{2}, \cdots$. Because of the convergence of the $\epsilon$ 's, $f(P)$ is uniquely determined. In order to see that the mapping $f$ is a homeomorphism of $E_{3}$ onto itself, let $P$ and $Q$ be any two distinct points in $E_{3}$. For some positive integer $m, d(P, Q)>1 / m$. Then for all $j>m$ we have by (1) above, $\epsilon_{j} \leqq \alpha_{m} / 3 \cdot j^{2}<d\left(P^{m}, Q^{m}\right) / 3 \cdot j^{2}$. Hence $\sum_{j=m+1}^{\infty} \epsilon_{j}<d\left(P^{m}, Q^{m}\right) / 3 \sum_{j=m+1}^{\infty} 1 / j^{2}$, and

$$
d\left(P^{m}, Q^{m}\right)>\sum_{j=m+1}^{\infty} 3 \epsilon_{j} .
$$

By Lemma 5.2, since $P$ and $Q$ were chosen arbitrarily in $E_{3}$, condition (2) is 
sufficient to prove that $f$ is a homeomorphism of $E_{3}$ onto itself.

It remains only to show that $f(M) \subset M_{3}^{1}$. For each $j$ we have $d\left(M^{j}, L_{j}\right)$ $=d_{j}>0$. Also $d\left[P^{j}, f(P)\right] \leqq \sum_{i=j+1}^{\infty} \epsilon_{i} \leqq \sum_{i=j+1}^{\infty} d_{j} / 3 \cdot i^{2} \leqq d_{j} / 3$. Then for $P$ in $M$, in particular,

$$
\begin{aligned}
d\left[L_{j}, f(P)\right] & \geqq d\left(L_{j}, P^{j}\right)-d\left[f(P), P^{j}\right] \\
& \geqq d_{j}-\frac{d_{j}}{3}>0 .
\end{aligned}
$$

Hence $f(M) \cap L_{j}=0$ for $j=1,2, \cdots$ Thus $f(M) \subset M_{3}^{1}$, and the proof of Theorem 5.5 is complete.

With slight modifications the method of proof of Theorem 5.5 yields the following stronger result.

THEOREM 5.6. The set $M_{3}^{1}$ contains a strongly homeomorphic image of every point set in $E_{3}$ which is the union of a countable number of compact, 0-dimensional sets.

Proof. Suppose $M=\bigcup_{i=1}^{\infty} M_{i}$, where each $M_{i}$ is compact and 0-dimensional. As in the previous theorem, the method of proof is to define a sequence $\left\{f_{i}\right\}$ of homeomorphisms of $E_{3}$ onto itself in such a way that the sequential limit transformation (in the sense of Lemma 5.2) is a homeomorphism of $E_{3}$ which carries $M$ into $M_{3}^{1}$.

In this case it is not sufficient to treat each $L_{i}$ only once. Instead we return to each $L_{i}$ infinitely many times, each time freeing it from a finite number of the $M_{i}$, as follows: let $f_{1}$ free $L_{1}$ from $M_{1}, f_{2}$ free $L_{1}$ from $M_{2}, f_{3}$ free $L_{2}$ from $M_{1} \cup M_{2}, f_{4}$ free $L_{1}$ from $M_{3}, f_{5}$ free $L_{2}$ from $M_{3}, f_{6}$ free $L_{3}$ from $M_{1} \cup M_{2} \cup M_{3}$, and so on. We again take the $\epsilon_{j}$ sufficiently small to insure that once an $L_{p}$ has been freed from one of the $M_{i}$ the remaining $f_{j}$ cannot carry a point of that $M_{i}$ back onto the $L_{p}$.

The proof of the existence of the $\alpha$ 's here also differs from that of Theorem 5.5 , because in this case $M$ is not necessarily contained in a finite cube in $E_{3}$. It can be shown, however, by consideration of a monotone increasing sequence $\left\{C_{i}\right\}$ of cubes that numbers $\left\{\alpha_{j}\right\}$ having the required properties do exist. At the $m$ th step we let $C_{m}$ be a cube with center at the origin which contains not only the $M$ 's being freed from an $L_{p}$ at that step but also all the preceding $C_{i}$.

With these exceptions the proofs of Theorems 5.5 and 5.6 are the same.

It is not known whether there is a zero-dimensional subset of $E_{3}$ which contains a strongly homeomorphic image of every compact zero-dimensional subset of $E_{3}$. It is possible, however, to show by an example that the set $M_{3}^{0}$ does not have this property. This leads one to suspect that the answer to the question posed at the beginning of this section is probably in the negative for the general case. 
EXAmple. Antoine's well known inner limiting set of an infinite sequence of chains of tori $\left[1\right.$, p. 663] is a compact, 0 -dimensional set $X$ in $E_{3}$ which has the property that any plane separating two points of $X$ in $E_{3}$ must intersect $X$. Clearly any strongly homeomorphic image of $X$ must also have this property. Suppose then that there is a homeomorphism $f\left(E_{3}\right)=E_{3}$ which carries $X$ into a subset of $M_{3}^{0}$. Then $f(X) \cap L_{3}^{1}=0$. But there is a rational plane which separates two points of $f(X)$, and this plane would necessarily intersect $f(X)$. This involves a contradiction, since every rational plane is contained in $L_{3}^{1}$.

\section{BIBLIOGRAPHY}

1. L. Antoine, Sur la possibilité d'étendre l'homéomorphie de deux figures à leur voisinage, C. R. Acad. Sci. Paris vol. 171 (1920) pp. 661-663.

2. A. I. Flores, Über $n$-dimensionale Komplexe die im $R_{2 n+1}$ absolut selbstverschlungen sind, Ergebnisse eines Mathematischen Kolloquiums vol. 6 (1933) pp. 17-24. 168.

3. M. Fréchet, Les dimensions d'un ensemble abstrait, Math. Ann. vol. 68 (1910) pp. 145-

4. W. Hurewicz and H. Wallman, Dimension theory, Princeton, 1941.

5. B. Knaster, Un continu dont tout sous-continu est indécomposable, Fund. Math. vol. 3 (1922) pp. 247-286.

6. G. Nöbeling, Über eine n-dimensionale Universalmenge im $R_{2 n+1}$, Math. Ann. vol. 104 (1930) pp. 71-80.

7. R. L. Moore, Foundations of point set theory, Amer. Math. Soc. Colloquium Publications, vol. 13, New York, 1932.

8. J. H. Roberts, Concerning topological transformations in $E_{n}$, Trans. Amer. Math. Soc. vol. 34 (1932) pp. 252-262. 328.

9. A. Schoenflies, Beiträge zur Theorie der Punktmengen, Math. Ann. vol. 62 (1906) pp. 286-

\section{Wittenberg College,} SPRINGFieLd, OHIO. 